\title{
INVARIANTS OF GRAPHS IN THREE-SPACE
}

\author{
LOUIS H. KAUFFMAN
}

\begin{abstract}
By associating a collection of knots and links to a graph in threedimensional space, we obtain computable invariants of the embedding type of the graph. Two types of isotopy are considered: topological and rigid-vertex isotopy. Rigid-vertex graphs are a category mixing topological flexibility with mechanical rigidity. Both categories constitute steps toward models for chemical and biological networks. We discuss chirality in both rigid and topological contexts.
\end{abstract}

\section{INTRODUCTION}

This paper introduces methods for producing topological invariants of graphs embedded in three-dimensional space. For this study, two types of graphs are considered: graphs with rigid vertices and graphs with nonrigid vertices. Our methods are strongest for graphs with rigid vertices.

The primary method in either case is to associate a collection of knots and links to the graph such that, for the appropriate notion of isotopy, this collection (the isotopy class of the collection) is an invariant of the isotopy type of the graph. One may proceed purely geometrically, or apply knot theoretic invariants to the collection.

This method is simple and powerful. By not opting too early for the extra structure of a polynomial invariant, we can often directly use the topology to create a minimal solution.

Nevertheless, it turns out to be particularly fruitful to use the new polynomial invariants of Jones and others (see $[3,5,6,7,9,10,13])$. Also, by combining knot theoretic invariants, we [10] have produced nontrivial graph-polynomial invariants in the case of 4-valent rigid vertex graphs. The latter invariants generalize the known two-variable polynomial invariants of knots and links (Homfly and Kauffman polynomials). These generalizations are joint work of the author and Pierre Vogel [10]. I shall show here $(\S 4)$ how these polynomials are related to the point of view involving a collection of links associated with the graph.

The paper is organized as follows:

In $\S 2$ we define ambient isotopy for nonrigid (topological) vertices and prove that piecewise linear ambient isotopy is generated diagrammatically by a set of moves (Figure 1) that generalize the Reidemeister moves). $\S 2$ then shows

Received by the editors December 1, 1987.

1980 Mathematics Subject Classification (1985 Revision). Primary 57M25. 
how to obtain first-order invariants of these topological graph moves. $\S 3$ works with rigid vertices and discusses the use of link polynomials in the category of rigid vertex four-valent graphs. Diagram-moves are given for rigid vertex isotopy. $\S 4$ discusses the relationship between the methods of this paper and other approaches that would produce direct polynomial invariants of links.

A similar invariant for rigid vertex graphs has been independently obtained by Ken Millett and David Jonish. We thank them for bringing this work to our attention. Work on this paper has been motivated by the possible application of these methods to problems in chemistry and molecular biology. We thank Jon Simon, Dennis Roseman, Keith Wolcott, David Walba, and DeWitt Sumners for stimulating conversations. Thanks go also to Cameron Gordon and John Conway for their beautiful paper [2] showing that every embedding of the complete graph on seven vertices contains a knot. This result and their technique form the inspiration for the present paper.

This work was partially supported by ONR Grant No. N0014-84-K-0 and NSF Grant No. DMS-8701772.

\section{REIDEMEISTER MOVES FOR GRAPHS}

Here I discuss (piecewise linear) ambient isotopy for graphs embedded in three-space.

A graph is a one-dimensional complex consisting of finitely many (zerodimensional) vertices and finitely many (one-dimensional) edges and loops. Each edge is homeomorphic to a closed line segment, and its ends form two distinct vertices in the graph. A loop is obtained by identifying the two endpoints of a segment; the point of identification is a vertex of the graph. We speak of edges in referring to either edges or loops. It is assumed that a graph is connected unless stated otherwise.

Embeddings of a graph are taken to be tame (equivalently, piecewise linear). In Theorem 2.1 I prove that ambient isotopy of embeddings for a graph in space is generated by a set of local moves that generalize the Reidemeister moves [ 15 and 16] for diagrams of knots. (These moves for graphs were first announced in [7].) Figure 1 illustrates the moves. Note that we have added to the usual list of Reidemeister moves two moves involving a vertex (moves IV and V). Move IV allows an edge to slide under or over a bundle of strands at a vertex. Move $\mathrm{V}$ allows any two adjacent (in the planar diagram) strands at a vertex to twist around one another. This is the basic topological vertex move-two strands at the vertex can twist without affecting the other strands.

With respect to these moves it is assumed that the embedding of the graph is presented as a planar diagram where each vertex neighborhood is a collection of rays emanating from the vertex. Crossings are transverse double points (as in knot and link diagrams) occurring between interior points of the edges. Such a diagram always exists via point projection from a representative in the ambient isotopy class of the graph. 


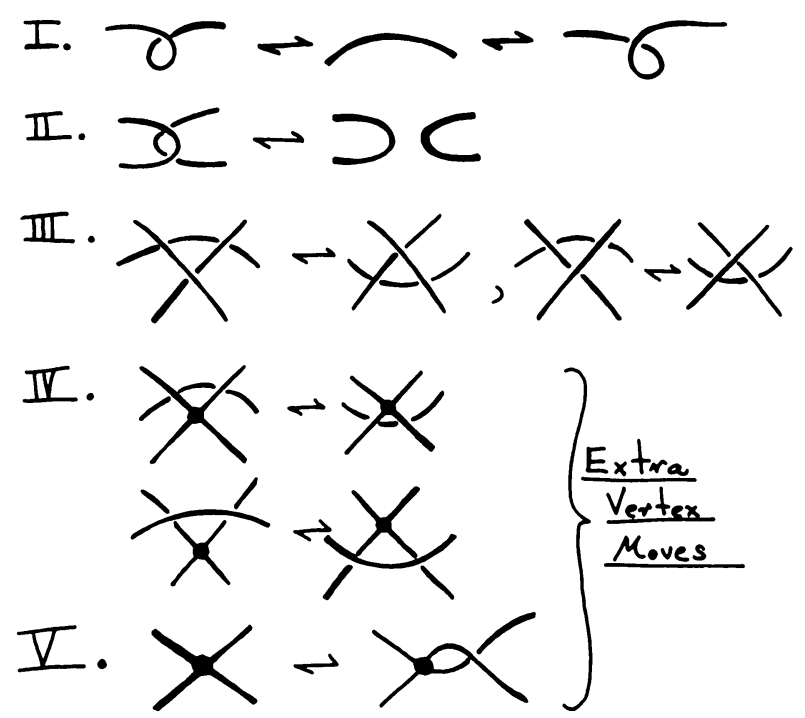

FIGURE 1. Reidemeister moves for graphs (topological vertex-valence four shown)

Theorem 2.1. Piecewise linear $(p l)$ ambient isotopy of embedded graphs is generated by the moves shown in Figure 1. That is, if two embedded graphs are ambient isotopic, then any two diagrams of them are related by a finite sequence of the moves of Figure 1.

Remark. The moves allow us to conceptualize the graph as an entity whose abstract graphical structure is invariant under the isotopy. For working with the graph as a piecewise linear complex, I assume that the embeddings of its edges are piecewise linear in Euclidean three-space. This means that a given embedding of $G$ is specified by the image of a finite set of points (the selected points, $I(G)$ ) consisting in the vertices of $G$ plus certain interior points of the edges of $G$ ). Two such points along an edge are said to be adjacent if there are no other selected points between them. The embedding is produced by extending straight line segments between adjacent selected points. It is assumed that the embedded interiors of all such segments are disjoint from one another and that the set of selected points $I(G)$ is mapped one-to-one into three-space. It is sometimes useful to think of the set of selected points as giving a structure to $G$ as a piecewise linear graph in three-space whose vertices are these selected points, and whose edges are all straight segments in space. This graphical structure is not invariant under ambient isotopy. In fact it follows from [4] that, just as in the case of pl embeddings of knots and links, that two pl graph embeddings are combinatorially isotopic (in the sense of Reidemeister [15]-to be explained below) if and only if one can be transformed into the other by an orientation preserving piecewise linear mapping of $R^{3}$ onto itself. Combinatorial isotopy is generated by elementary combinatorial isotopies as illustrated in Figure 2. In an elementary isotopy a triangle is embedded piecewise linearly in $R^{3}$ so that 


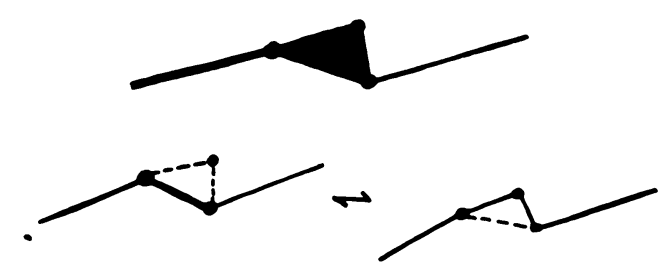

FIGURE 2. Elementary combinatorial isotopy

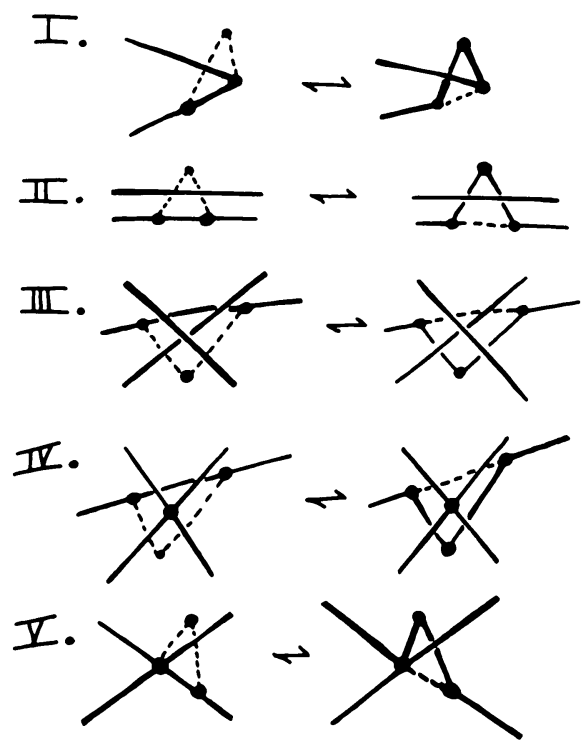

FIGURE 3. Reidemeister moves are elementary combinatorial isotopies

its interior is disjoint from the graph embedding, and so that the triangle shares either one or two edges with the embedded graph (in the sense of the piecewise linear edges generated by the selected points). In an elementary combinatorial isotopy the shared edges of the triangle are replaced by the unshared edges. The result is a new $\mathrm{pl}$ embedding of the same graph with a different set of selected points.

The paper [4] was the first to prove that pl ambient isotopy and combinatorial isotopy are equivalent for $\mathrm{pl} \mathrm{links} \mathrm{in} \mathrm{three-dimensional} \mathrm{space.} \mathrm{This} \mathrm{provides} \mathrm{the}$ appropriate context for Reidemeister's theory of moves. Reidemeister showed how combinatorial isotopy implicates the diagrammatic equivalence of knots and links generated by the three Reidemeister moves [15]. The technique of [4] shows that Reidemeister's notion of combinatorial equivalence was identical to piecewise linear ambient isotopy. These techniques go directly over for the extension to embeddings of graphs. Thus in order to prove Theorem 2.1 it will suffice for us to show that the generalized Reidemeister moves of Figure 1 can generate combinatorial isotopy (via triangle moves) for pl embedded graphs.

A historical remark is in order here. At the time when Reidemeister wrote his book [15] the different equivalence relations and piecewise linear techniques 


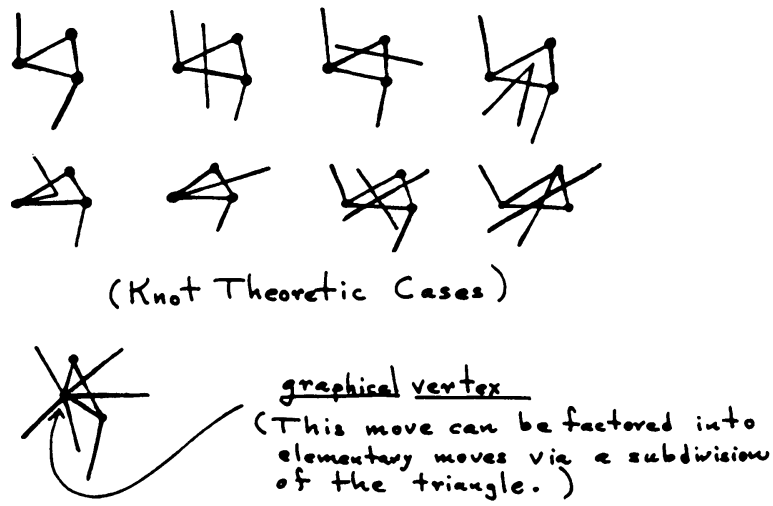

FIGURE 4. Examples of triangle in relation to graph projection
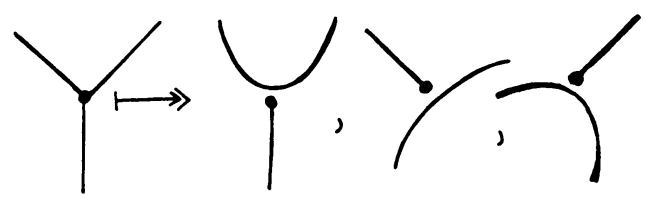

FIGURE 5. Local replacements at a topological vertex (illustrated for valence three)

had not been fully sorted out. Reidemeister took combinatorial isotopy as the fundamental equivalence relation, and proved all his results on this basis. The intervening years have shown that his intuition was correct in this matter (for the theories of tame knots, links and graphs).

Proof of Theorem 2.1. In order to prove that the extended Reidemeister moves of Figure 1 suffice to generate combinatorial isotopy it is first necessary to formalize them to their corresponding piecewise linear versions as shown in Figure 3. Then the proof follows via an enumeration of all cases of a triangle (for the elementary move) in general projected position with respect to the piecewise linear diagram. Figure 3 in fact shows that each of the extended Reidemeister moves is an elementary combinatorial isotopy. Figure 4 shows examples of cases in the enumeration. Note that we can subdivide the triangle if so needed, and that elementary moves that do not change the crossing structure of the diagram are not included in the list of extended Reidemeister moves. I leave the full enumeration indicated in Figure 4 for the reader. This completes the proof of Theorem 2.1.

A first graph invariant. This invariant is a formalization of the notion of "all knots and links in the graph". A collection $T(G)$ of knots and links to the graph $G$ will be associated. These are obtained as follows: At each vertex make a local replacement of the type indicated in Figure 5.

This replacement leaves two edges connected and unplugs all the other edges at this vertex, leaving them as free ends. Since any two edges can be left connected at a vertex there are $N(N-1) / 2$ choices available for the replacement at a vertex of degree $N$. 

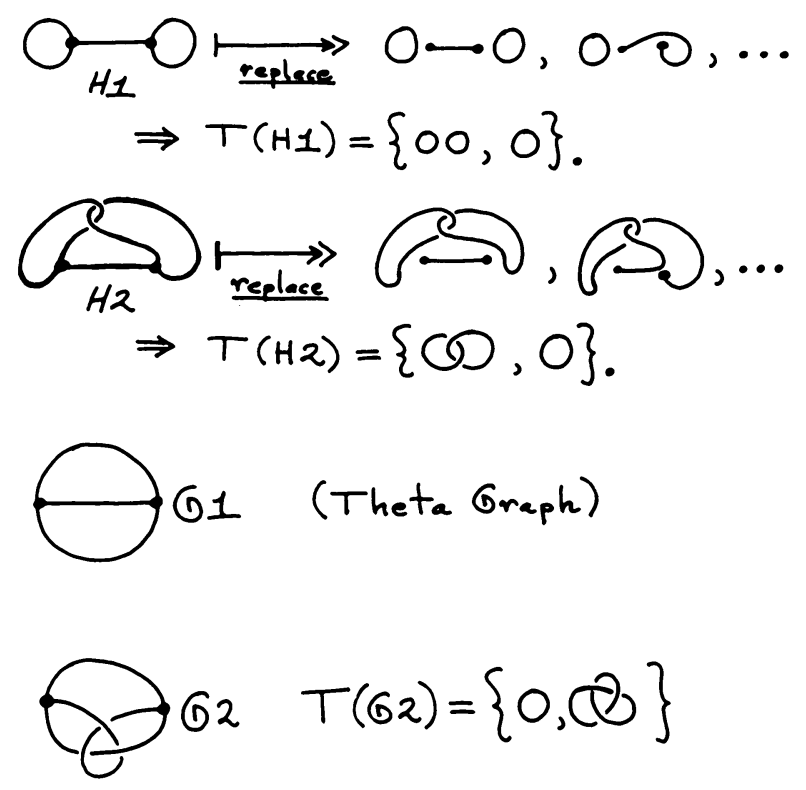

Figure 6

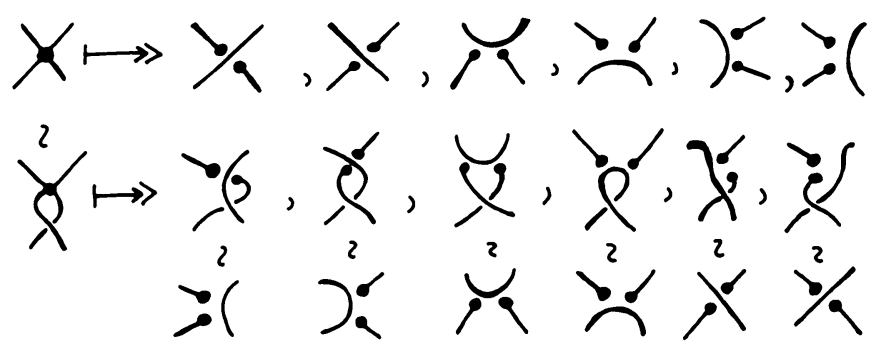

FiguRE 7. Braiding at a vertex permutes the replacements

Having chosen a replacement at each vertex of the graph, let $r(G)$ denote the link formed by the closed curves formed by this process. (Eliminate the unkotted arcs.)

Then $T(G)$ is the collection of links $r(G)$ for all possible replacement choices. See Figure 6 for examples of the formation of $T(G)$.

Theorem 2.2. Let $G$ be any graph embedded in three-dimensional space, and presented diagrammatically. Then the collection of knots and links $T(G)$, taken up to ambient isotopy, is a topological invariant of $G$.

Proof. Examine each of the elementary moves, and note that, up to ambient isotopy, the elements of the collection $T(G)$ are simply permuted by the moves. (Compare Figure 7.) 


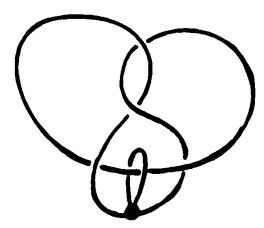

FIGURE 8. J. Simon's graph

Examples. Here I refer to the examples shown in Figure 6. The handcuff graph $H 2$ is not ambient isotopic to the planar $H 1$ because $T(H 2)$ contains a nontrivial link. The theta graph $G 2$ is not planar because $T(G 2)$ contains a trefoil knot. These same techniques show that the graph $G$ of Figure 8 is not planar, but they do not show (since the figure eight knot is archiral) that $G$ is not ambient isotopic to its mirror image. In the case of $G$, this has been shown by Wylbur Whitten using a more subtle approach using branched coverings. This is a case where the branched covering techniques (compare [17 and 20]) are definitely superior to simply looking at a list of associated knots and links.

The problem of constructing good invariants in the general case is quite difficult, due to the generation of arbitrary braiding at a vertex via the extended move number $\mathrm{V}$.

It is of interest to imagine physical situations in which the relation of combinatorial equivalence for graphs is relevant (compare [17]). For example, imagine that each vertex is replaced by a ball and that the edges incident to the vertex are strings attached to the surface of the ball by magnets that are free to move over the surface. Then it is clear that the braiding at the vertex corresponds to configurational movements of the magnets on the surface. If, instead, the vertex is replaced by a disk to which the strings are rigidly attached, we shall have the case of the rigid vertex as described in the next section.

The main point of this section has been to point out that there are Reidemeister moves for embedded graphs, just as for knots, and to point out that these moves are justified on the same basis as the case of knots and links. This reformulates the general problem of the classification of piecewise linear graph embeddings to the study of a diagrammatic formal system whose equivalence relation is generated by the extended Reidemeister moves. This system is particularly important not only because it is very challenging to produce good combinatorial invariants, but also because it distills the problem of $\mathrm{pl}$ graph embedding into a managable formalism, just as do the diagrams and moves of the theory of knots.

\section{RIGID VERTICES}

I now specialize to 4-valent graphs with rigid vertices.

Physically, the concept of rigid vertex corresponds to a network whose vertices are rigid disks each having (four) flexible tubes or strings emanating from 
it. Since the disk is rigid, it turns as a whole when rotated by 180 degrees, and it twists the strings in pairs as shown in Figure 9.

In terms of diagrammatic moves, the concept of rigid vertex involves the replacement of move $\mathrm{V}$ of Figure 1 by the move $\mathrm{V}^{*}$ shown in Figure 9.

The rigidity of the vertex forces double braiding when the vertex is turned by 180 degrees. Also the second move (with a three strand twist) under $\mathrm{V}^{*}$ can be accomplished up to ambient isotopy by the first (paired twist). Thus we only need consider one extra braiding move.

We define rigid vertex equivalence (just called equivalence in this section) to be the equivalence relation generated by the moves I, II, III, IV, ${ }^{*}$. A 4-valent rigid vertex graph will be referred to as an RV4 graph.

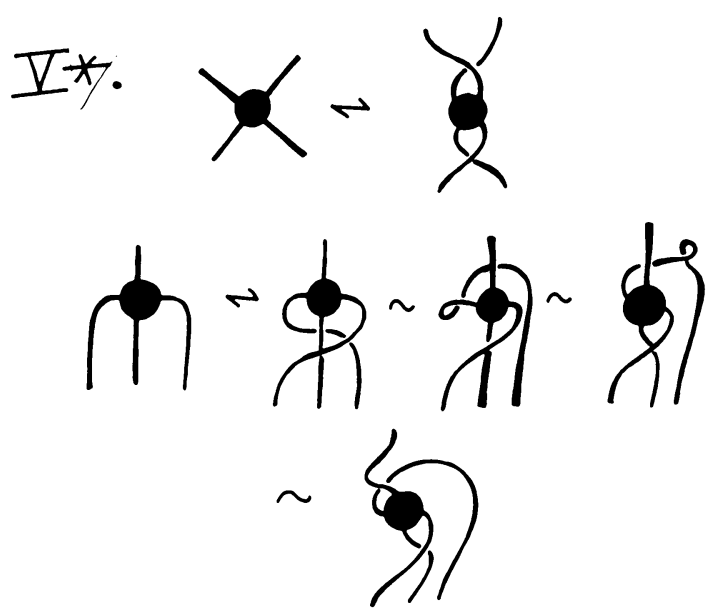

FIGURE 9. Braiding move for rigid vertex (corresponds to $180^{\circ}$ twist)

Remark. One needs a repeat, in this context, of the discussion of $\S 2$, where combinatorial equivalence generated by extended Reidemeister moves is seen to generate pl ambient isotopy of embedded graphs. In the case of rigid vertices of arbitrary valence this is done by replacing each vertex by a polygon so that each graph-vertex strand (corresponding to an edge that emanates from this vertex of the graph) emanates from one of the vertices of the polygon. Then the resulting complex is allowed to undergo pl ambient isotopy, except that the polygons must be moved rigidly (i.e. by an orientation preserving orthogonal transformation or a length preserving affine transformation of three-space). Such ambient isotopies can be generated by combinatorial isotopies that involve no triangle expansions or contractions on the polygonal vertices-along with rotations of the polygons. (As a polygon rotates the pl strands attached to it undergo sequences of combinatorial isotopies.) At the diagrammatic level, the rotations of the polygons reduce to 180 degree turns of the polygons that turn their attached strings as in move $\mathrm{V}^{*}$. With this scheme, the arguments of 

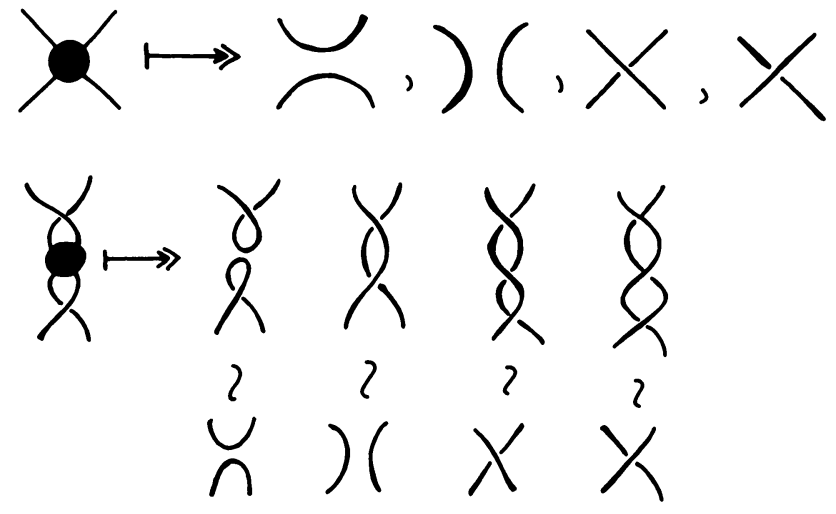

FIGURE 10. Rigid vertex connection replacements

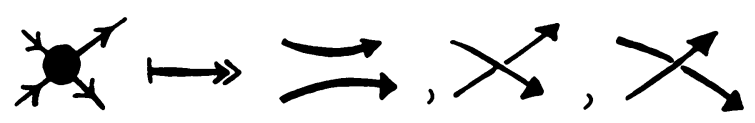

FIGURE $10^{\prime}$. Oriented rigid vertex replacements

$\S 2$ extend to show that pl ambient isotopy of rigid vertex graphs is generated by the moves of Figure 9. There are other approaches to the concept of the rigid vertex. I have suggested regarding it as an actually rigid macroscopic object, hence replacing the graph by a cell complex with extra structure. The paper [1] is a good introduction to topological properties of embedded cell complexes in relation to graph-embeddings. In particular, Boyle gives geometric proofs of chirality for Möbius ladders shown chiral in [17] by branched covering techniques. His methods are likely to be of use in further articulation of the concept of a rigid vertex.

Remark. One of the interesting aspects of RV4 graphs under equivalence is that one can actually build simple physical models for them-just as one can form models for knots and links from rope or string. As the above remarks show, rigid vertex isotopy is a mixture of mechanical (geometrical) and topological elements. It is relatively easy to produce models-by attaching flexible strings or tubes to rigid disks or balls. The rigid isotopy is closely related to motions of networks in space that are a mixture of the mechanical and the topological.

Unoriented invariants of rigid vertex isotopy. We shall associate a collection of knots and links to each RV4 graph. The collection associated to a graph $G$ will be denoted $C(G)$.

An element of $C(G)$ is obtained by making a connection at each vertex, replacing the vertex locally by a configuration that connects the four edges in pairs. There are four ways to do this, as illustrated in Figure 10. 
Theorem 3.1. For an $R V 4$ graph $G$ the ambient isotopy class of $C(G)$ is an invariant of the rigid equivalence class of the graph $G$.

Proof. Observe the effect of the type $\mathrm{V}^{*}$ move as shown in Figure 10. This proves invariance for type $\mathrm{V}^{*}$. Invariance under the other moves is trivial.

This theorem is -quite powerful in analyzing RV4 graphs. Just as in the topological case, any invariants of knots and links can be applied to the elements of the collection $C(G)$. In this case we can use this information to decide subtle chirality questions in the rigid vertex context.

Theorem 3.2. Let $G$ be a 4-valent rigid graph embedded in three-space. Let $C(G)$ denote the collection of link diagrams associated with the diagram $G$. Call an element $K$ of $C(G)$ singularly chiral if it is chiral, and no other element of $C(G)$ is ambient isotopic (link-theoretic ambient isotopy) to the mirror image of $K$. If any element of $C(G)$ is singularly chiral, then the graph $G$ is chiral in the rigid vertex category.

Proof. This result follows at once, since the list $C\left(G^{\prime}\right)$ for the mirror image diagram $G$ consists in the mirror images of all elements of the list $C(G)$.

Example. This example continues the discussion of the graph $G$ of Figure 8 . Looking at it from the rigid vertex point of view we see from Figure 11 that $G$ is certainly not RV4 planar. Furthermore, it is not RV4 equivalent to its mirror image. For if $G$ where achiral, then the individual knots and links in $C(G)$ (being distinct) would each be achiral. We then check that this is not the case by using our results for the Jones polynomial of alternating knots.

Remark. It is the purpose of this example to show how the new polynomial invariants can be used in this context. Thus, while chirality can be verified in this case by other techniques, the bracket version of the Jones polynomial [6] provides a particularly elementary way to proceed. Furthermore, the technique

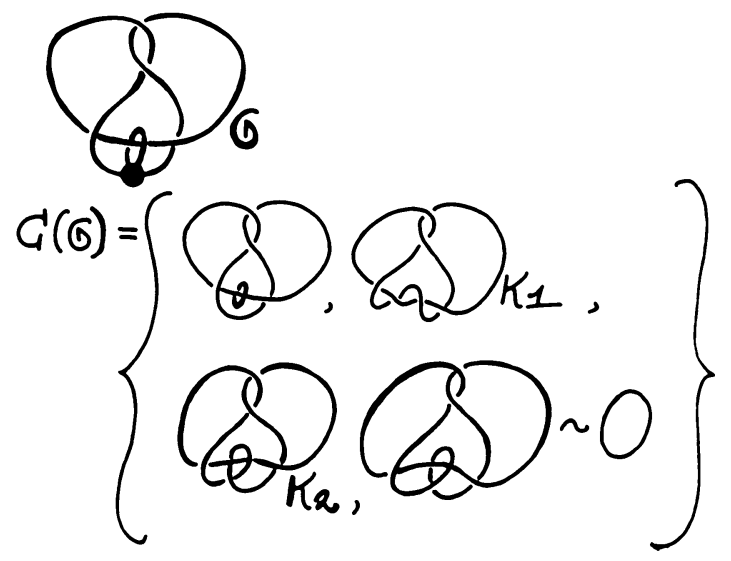

FIGURE 11 


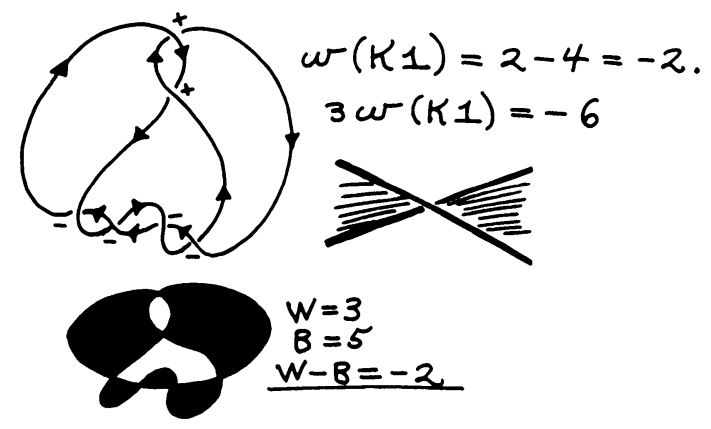

FIGURE 12

is quite general, and allows the use of the computer programs that calculate these polynomials.

In the case at hand, we know from [6] that if $K$ is achiral alternating then $3 w(K)=W-B$ where $w(K)$ is the twist number of $K$ and $W$ and $B$ are the numbers of white and black regions in a shading where all crossings are shaded as shown in Figure 12. Choosing $K 1$ in $C(G)$, we find as shown in Figure 12 that this equation is not satisfied. Hence $K 1$ is chiral. Hence $G$ is RV4 chiral. (The twist number is the sum of the crossing signs, as illustrated in Figure 12.)

As a second example, consider the graph $G^{\prime}$ shown in Figure 13. $G^{\prime}$ is obtained from $G$ of Figure 11 by switching two crossings. To prove $G$ not RV4 equivalent to $G^{\prime}$ it suffices to show that $K 2^{\prime}$ (Figure 13) is not ambient

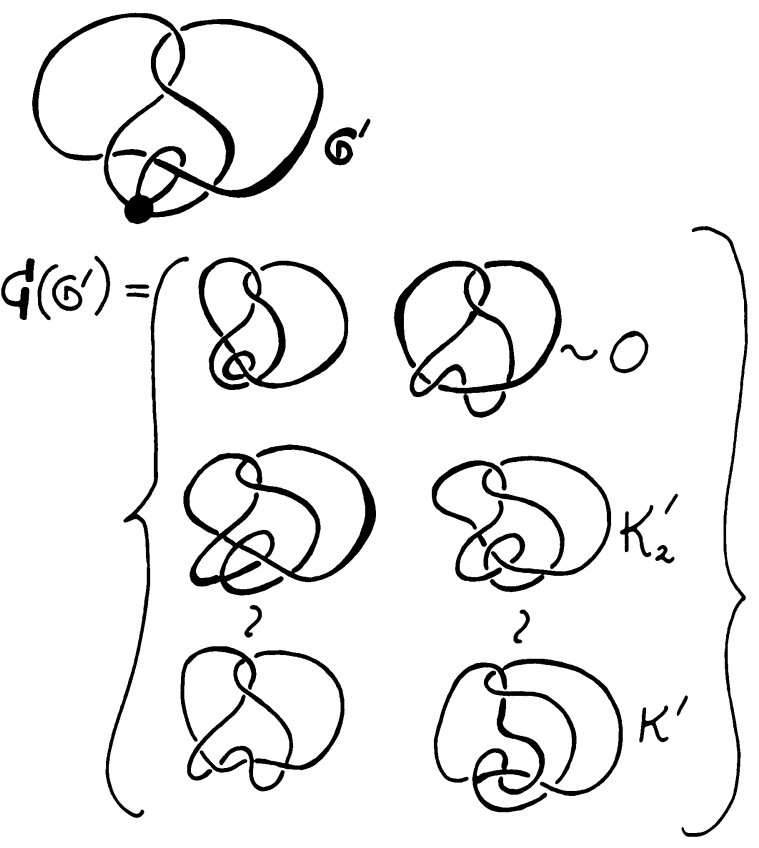

FIGURE 13 
isotopic to $K 2$ (Figure 11). It follows from the theorems about alternating knots in [6,14 and 19] that a nonalternating diagram sharing the same (reduced) underlying planar shadow as an alternating knot cannot be ambient isotopic to the alternating knot. Therefore $K 2$ and $K 2^{\prime}$ are distinct. Hence $G$ and $G$ are not RV4 equivalent.

To verify that $G$ is RV4 chiral, it is sufficient to know the chirality of $K 2^{\prime}$. As shown in Figure 13, the knot $K 2^{\prime}$ is ambient isotopic to an alternating knot $K^{\prime}$, and the same check as above (using white and black region counts) shows that $K^{\prime}$ is chiral.

One final example: The rigid vertex graph shown in Figure 14 is chiral (as our technique easily shows). As a topological vertex graph it is achiral. This underlines the differences between these two categories.

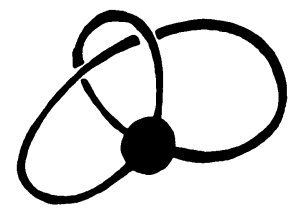

FIGURE 14

Oriented invariants of rigid vertex isotopy. Everything that we have said in the unoriented case goes over with obvious modification to the oriented case. View Figure $10^{\prime}$. This illustrates the replacements for an oriented rigid vertex. The same arguments as before show that the associated set of oriented links $C(G)$ for an oriented graph $G$ is an ambient isotopy invariant of $G$. I will not go into further details in this case.

\section{POLYNOMIAL INVARIANTS OF EMBEDDED GRAPHS}

There are now a number of methods available for creating polynomial invariants of rigid vertex graph-embeddings [10,12 and 21]. I shall restrict my comments here to the case of 4 -valent vertices.

In this case of RV4 graphs, the approach explained in the present paper may be just as powerful as the available polynomial invariants.

The reason for this is very simple. The polynomial invariants are constructed by adding up link polynomials of the elements of $C(G)$ (the list of links associated with the graph $G$ ), multiplied by appropriate coefficients. If the link polynomials applied to the individual elements of $C(G)$ do not detect chirality for any element of $C(G)$, then in order for the resulting summation polynomial to detect chirality, an asymmetry in the polynomial will have to arise from some subtlety in the summation.

This leads to the problems:

1. Give an example of an RV4 chiral graph whose chirality is not detected from the list of associated links $C(G)$. 
2. Give an example of an RV4 chiral graph whose chirality is not detected by the list of associated links $C(G)$, but so that its chirality is detected by a graph-polynomial.

Since I do not know an example for question number 1, I do not yet know examples for either of these questions. Both questions underscore the rather remarkable character of the rigid vertex category-that much information can be gained at very little cost by simply looking at all the knots and links in the graph.

For the reader unfamiliar with graph-polynomials, here is a description of the Kauffman-Vogel polynomial in the unoriented (Dubrovnik) case. This polynomial generalizes the graph polynomial of Yamada [21] for the RV4 case (unoriented). It is a three variable generalization of the two-variable Kauffman (Dubrovnik) polynomial [9]. The polynomial satisfies the equations:

$$
\begin{aligned}
& {[z-A]=A[=]+B[)(]+[X]} \\
& {[X]=B[\overline{=}]+A[)(]+[X]} \\
& {[\partial-]=a[-],[-\sigma]=a^{-1}[-]}
\end{aligned}
$$

where the small diagrams stand for larger diagrams that are identical except for the indicated differences. This polynomial is a polynomial in the three variables $A, B$ and $a$. With $Z=A-B$, we see that

$$
[X]-[X]=z([\Sigma]-[)(])
$$

Thus $[\mathrm{K}]$ specializes to the regular isotopy (see [7 or 8 or 9$]$ ) version of the Dubrovnik polynomial (see [9 or 10]) in the case where $K$ has no graphical vertices. (For $a=1$, the Dubrovnik polynomial is a trivial invariant. A corresponding substitution in the Kauffman polynomial yields the $Q$-polynomial of Brandt, Lickorish, Millett and Ho. See [9 and 13].)

$[G]$ is defined for graphs by expanding away the graphical vertices via the formulas (rewritten from above):

$$
\begin{aligned}
& {[X]=[\chi]-A[=]-B[)(]} \\
& {[X]=[K]-B[=]-A[)(] .}
\end{aligned}
$$

It is not hard to see that the well-definedness of the Dubrovnik polynomial implies that this graph-polynomial is a well-defined invariant of rigid-vertex isotopy up to multiplication of the entire polynomial by powers of $a$.

The polynomial for a mirror image is obtained by interchanging $A$ and $B$ and replacing $a$ by $(1 / a)$. 
While the Kauffman-Vogel generalizations of the known two-variable polynomials for links may not do more for RV4 graphs than the general method of this paper, these generalizations and the corresponding methods of Yamada, Millett and Jonish shed light on the relationship of rigid vertex equivalence and the meaning of the new polynomial invariants.

Furthermore, an unknown variation on these themes could very well lead to new information. The possibility for new invariants in this domain is very real.

\section{REFERENCES}

1. J. Boyle, Embedings of 2-dimensional cell complexes in $S^{3}$ determined by their 1-skeletons, (preprint).

2. J. H. Conway and C. McA. Gordon, Knots and links in spatial graphs, J. Graph Theory 7 (1983), 445-453.

3. P. Freyd, D. Yetter, J. Hoste, W. Lickorish, K. Millett and A. Ocneanu, A new polynomial invariant of knots and links, Bull. Amer. Math. Soc. 12 (1985), 239-246.

4. W. Graeub, Die semilinearen abbildungen, S. B. Heidelberger Akad. Wiss. Math.-Nat. Kl. (1950), 205-272.

5. V. F. R. Jones, A new polynomial invariant for links via von Neumann algebras, Bull. Amer. Math. Soc. 12 (1985), 103-112.

6. L. H. Kauffman, State models and the Jones polynomial, Topology 26 (1987), 395-407.

7. __ New invariants in knot theory, Amer. Math. Monthly 95 (1988), 195-242.

8. __ On knots, Ann. of Math. Studies, no. 115. Princeton Univ. Press, 1987.

9. __ An invariant of regular isotopy, Trans. Amer. Math. Soc. (to appear).

10. L. H. Kauffman and P. Vogel, Link polynomials and a graphical calculus (preprint 1987).

11. R. Litherland, The Alexander module of a knotted theta-curve (to appear).

12. K. Millett, Stereotopological indices for a family of chemical graphs, J. Comput. Chem. 8 (1987), 536-548.

13. K. Millett, B. Brandt and W. B. R. Lickorish, A polynomial invariant for unoriented knots and links, Invent. Math. 84 (1986), 563-573.

14. K. Murasugi, Jones polynomials and classical conjectures in knot theory, Topology 26 (1987), 187-194.

15. K. Reidemeister, Knotentheorie, Ergebnisse der Matematik und ihrer Grenzgebiete, (Alte Folge), Band 1, Heft 1, Springer 1932; reprint, Springer-Verlag, 1974.

16. __ Knot theory, Translated into English by L. Boron, C. Christenson, and B. Smith, Univ. of Idaho, Moscow, Idaho, BCS Associates, 1983.

17. J. Simon, Topological chirality of certain molecules, Topology 25 (1986), 229-235.

18. __. Molecular graphs as topological objects in space, J. Comput. Chem. 8 (1987), 718-726.

19. M. Thistlethwaite, A spanning tree expansion for the Jones polynomial, Topology 26 (1987), 297-310.

20. K. Wolcott, The knotting of theta curves and other graphs in $S^{3}$, Thesis, Univ. of Iowa, 1985.

21. S. Yamada, An invariant of spatial graphs, (preprint 1987).

Department of Mathematics, Statistics and Computer Science, The University of Illinois at Chicago, Chicago, Illinois 60680

Current address: Institut des Hautes Etudes Sciences, 91440 Burs-sur-Yvette, France 